\title{
PEDIATRIC UROLOGY
}

\section{Does the less aggressive multimodal approach of treating bladder-prostate rhabdomyosarcoma preserve bladder function?}

\author{
Soler R, Macedo A Jr, Bruschini H, Puty F, Caran E, Petrilli A, Garrone G, Srougi M, Ortiz V \\ Department of Urology, Federal University of Sao Paulo, Sao Paulo, Brazil \\ J Urol. 2005; 174: 2343-6
}

Purpose: The treatment of bladder-prostate rhabdomyosarcoma has evolved into multimodal therapy, including chemotherapy, radiotherapy and organ sparing surgery with bladder preservation. We investigated bladder function in children who underwent multimodal therapy at our institution and retained the original bladder for at least 6 months after treatment ended.

Materials and Methods: We evaluated 8 children with bladder-prostate rhabdomyosarcoma treated at our institution between 1999 and 2003 according to inclusion criteria. All patients underwent history, physical examination and urodynamic study at least 6 months after completion of treatment (range 6 to 39 months).

Results: All patients were treated following the same chemotherapy and radiotherapy scheme. Three patients $(37.5 \%)$ were asymptomatic and had normal urodynamic studies, and 1 had only dysuria (this patient later underwent continent urinary diversion with transverse colon). The 4 remaining patients had urological complaints, and the urodynamic findings were reduced bladder capacity in 4 , overactivity plus sensory urgency in 2 , sensory urgency only in 1 and suprapubic pain during filling in 1.

Conclusions: Among 8 patients 3 had normal urinary function and 4 had minor tolerable alterations. Cystectomy and urinary diversion were later necessary in only 1 patient due to disabling dysuria. The fact that the original functioning bladder was preserved in 7 of 8 patients suggests the feasibility of multimodal therapy. Long-term followup will still be necessary for definite conclusions, since we recognize that the deleterious effects, mainly of radiotherapy, may take longer to become evident.

\section{Editorial Comment}

The authors review their experience with an organ-sparing approach to treatment of pelvic rhabdomyoscarcoma in your children. Eight patients were reported from those treated between 1999 and 2003. The results were very encouraging, with only one patient requiring cystectomy during follow-up for dysuria.

Although high cure rates are possible with aggressive therapy that includes surgical extirpation of the bladder, efforts in recent years have focused on obtaining the same cure rates while preserving the bladder. Data on whether this can be done successfully are somewhat limited. This report is an important follow-up that provides valuable data for the clinician facing a new patient with this problem.

Despite the positive message however, there are several caveats. First, two patients were excluded. One had an early recurrence (we do not know whether this patient survived) and another is reported as having a "head injury." Bladder preservation at the expense of survival may not be the best outcome. Second, perhaps even more important, only 3 of the 8 patients were asymptomatic at the time of follow-up, which is admittedly short. Will they stay asymptomatic? What about the 4 remaining with their bladders? Will they develop increasing symptoms over time? Will they ultimately require a cystectomy? Longer follow-up is needed. Despite these questions, the report provides promising and important information.

Dr. Barry A. Kogan

Chief and Professor of Urology and Pediatrics Albany Medical College Albany, New York, USA 


\section{Small intestinal submucosa bladder neck slings for incontinence associated with neuropathic bladder Misseri R, Cain MP, Casale AJ, Kaefer M, Meldrum KK, Rink RC Department of Pediatric Urology, James Whitcomb Riley Hospital for Children, Indiana University School of Medicine, Indianapolis, Indiana, USA J Urol. 2005; 174 (4 Pt 2): 1680-2; discussion 1682}

Purpose: We assess the results using small intestinal submucosa (SIS) for neuropathic urinary incontinence in a large single institutional experience. Ambulatory status was considered as a possible predictor of success.

Materials and Methods: We retrospectively reviewed the charts of patients treated with SIS bladder neck sling procedures for neuropathic urinary incontinence with a leak point pressure less than $25 \mathrm{~cm} \mathrm{H} 2 \mathrm{O}$ and a minimum of 6 months followup. Continence was defined as wet (requiring pads or diapers) or dry (requiring no pads and dry underwear). Patients were classified as ambulatory (able to ambulate without assistance or using braces, crawling at home) or nonambulatory (confined to a wheelchair). Results were analyzed with regard to patient sex, ambulatory status and simultaneous bladder neck repair.

Results: A total of 21 females and 15 males 3 to 10 years old (mean age 9 years) were treated with SIS bladder neck slings (sling alone 27, bladder neck repair with SIS sling 9). Slings were performed along with reconstructive surgery in all cases (all had creation of urinary catheterizable channels and simultaneous or prior bladder augmentations). Minimum followup was 6 months (mean 15, range 6 to 42). Overall, 27 of the 36 patients $(75 \%)$ are dry following bladder neck sling. In patients treated with the sling procedure alone 6 of 8 (75\%) nonambulatory females and 8 of $10(80 \%)$ ambulatory females were continent, and 3 of $4(75 \%)$ nonambulatory males and 2 of $5(40 \%)$ ambulatory males were dry.

Conclusions: SIS has equivalent rates of continence compared to series using rectus fascia in patients with neuropathic urinary incontinence. The ambulatory status of males should be considered when determining which treatment option is best for the patient with myelodysplasia and neuropathic sphincteric incontinence, as in our series ambulatory males undergoing sling placement alone had a poor outcome.

\section{Editorial Comment}

The authors report a series of 36 children with neurogenic bladder who underwent a bladder neck sling using small intestinal submucosal (SIS) as manufactured by Cook Urological. SIS has the advantage of being "off-the-shelf." In addition, in this neurogenic population, rectus muscle and fascia may not be normal and may be further weakened and scarred by harvesting it, making autologous rectus fascia a less favorable alternative. Overall, $75 \%$ of the authors' patients were dry afterwards.

In general, these results are good, but several important points need to be considered. For the reader, it is important to determine whether this success rate is due to the operative technique or the material being used. In reviewing the data, we do not know whether this is a consecutive series or whether these patients were selected for the SIS sling (and how they may have been selected). We do not know how many other types of slings were performed in the same or similar time period and we have no idea what the authors' success rates were with other materials or other techniques. Moreover, the 36 patients had widely varying characteristics, including gender, ambulatory status, history of/need for augmentation, and even the type of sling (with or without bladder neck surgery). The authors attempt some sub-group analyses and conclude that the operation works less well in ambulatory males. Though this may be true, it is difficult for the reader to determine this with any certainty in the large, varied group. Also, as in all studies of continence, how the continence is determined is of critical importance. In this case, it was reported by patients or their parents. However, it is not known how this was reported. For example, obtaining these data face-to-face with a provider has been shown to result in falsely elevated rates of continence, as the patient does not wish to disappoint the provider. 
Moreover, perhaps the biggest concern is with durability. Mean follow-up was only 15 months. Presumably, SIS acts as a template for the in-growth of other tissues. What other tissues grow in? How strong are they? How durable are they? Many stress incontinence procedures have been shown to not stand the test of time. The patients in this series are young and, because of their neurogenic status, they will probably have their continence tested more due to straining than non-neurogenic patients. Although these results are encouraging, they should be considered preliminary. Much longer follow-up is needed.

Dr. Barry A. Kogan Chief and Professor of Urology and Pediatrics Albany Medical College Albany, New York, USA 